\title{
Hyperechogenicity of Lenticular Nuclei in Primary Cervical Dystonia
}

\section{Agnieszka Krzak-Kubica ${ }^{*}$, Stanisław Ochudło ${ }^{1}$, Justyna Gawryluk', Krzysztof Pawlicki², Monika Rudzińska-Bar ${ }^{1}$}

${ }^{1}$ Department of Neurology, Medical University of Silesia, University Medical Center, Katowice, Poland

${ }^{2}$ Department of Biophysics, Medical University of Silesia, University Medical Center, Katowice, Poland

\begin{abstract}
Dystonia is one of the most common movement disorders, but the pathogenesis of primary dystonia is not fully understood. One of the proposed pathomechanisms is the deficiency or dysfunction of ATP-dependent Menkes protein responsible for the extracellular transport of copper within subcortical nuclei, the lack of which could lead to increased accumulation of copper in the lenticular nucleus (LN). In 1995, Naumann et al. performed transcranial sonography (TCS) on patients with dystonia for the first time. Other researchers confirmed more frequent occurrence of hyperechogenic LN more than $70 \%$ patients with cervical dystonia. The aim of our study was therefore to evaluate the prevalence of hyperechogenic changes in lenticular nucleus of the brain in patients with cervical dystonia. 108 patients were considered eligible for inclusion; 42 of them were subsequently excluded due to occurrence of exclusion criteria. Thus, the study involved 66 patients and 71 controls. Hyperechogenicity of the lenticular nucleus was found more often in patients with cervical dystonia than in controls $(p<0.0001)$. It was unilateral in majority of patients $(75.8 \%)$. The average surface area of hyperechogenic changes is bigger in dystonic patients compare with control group $0,05( \pm 0,10)$ vs 0,005 $( \pm 0,02)$ on the left side and $0,10( \pm 0,12)$ vs. $0.004( \pm 0.02)$ on the right side. Hyperechogenicity of lenticular nuclei were observed in the case of 8 patients $(11.2 \%)$ within the control group and they were unilateral changes. Substantia nigra hyperechogenicity did not differ between the two groups. We did not find a correlation between the size of the observed changes and dystonia severity or duration of the disease. The only correlation was the association between the width of the third ventricle and age of patients. We suggest that the hyperechogenic changes within the lenticular nuclei are prevalent in patient with primary cervical dystonia and are an important diagnostic clue.
\end{abstract}

Keywords: Transcranial sonography; Lenticular nucleus; Primary cervical dystonia

\section{Introduction}

Dystonia is one of the most common movement disorders in which abnormal, repetitive muscle contractions occur, frequently causing twisting movements or incorrect body posture [1]. The pathogenesis of primary dystonia is not fully understood. Although neuropathologic studies did not reveal consistent abnormalities, electrophysiologic and neuroimaging findings point towards disinhibition and overactivity of the frontal motor cortical areas caused by an altered basal ganglia outflow $[2,3]$. The probable cause is the dysfunction within neuronal circuits involving subcortical nuclei, thalamus, motor cortex and cerebellum [4-7]. The relative contribution of specific neurotransmitters and pathways is intensively studied but has not been established yet [8-11]. One of the proposed pathomechanisms is the deficiency or dysfunction of ATP-dependent Menkes protein responsible for the extracellular transport of copper within subcortical nuclei, the lack of which could lead to increased accumulation of copper in the lenticular nucleus [12-14]. Accordingly, decreased levels of mRNA for Menkes protein and reduced copper concentrations were found in peripheral blood lymphocytes of patients with cervical dystonia [15]. Other hypotheses emphasize the effect of concomitant moderate accumulation of manganese $[13,16]$.

Primary dystonia is diagnosed based on medical history, neurological examination and lack of observable brain abnormalities on neuroimaging [17]. Specificity of diagnosis is impeded, however, by the lack of biological marker. The usefulness of transcranial sonography (TCS) in the detection of structural abnormalities within basal ganglia was shown for the first time in 1995, when Becker et al. [18] reported on specific abnormalities within substantia nigra of patients with Parkinson disease (PD). Neuman [4] was the first to report ultrasonographic changes of lenticular nucleus in idiopathic dystonia in 1996. Since then, TCS of subcortical nuclei has become an active field of research. Based on previous literature and present results we now hypothesize that LN hyperechogenicity may be an ultrasound feature of all cervical dystonia.
The objective of our study was therefore to evaluate the prevalence of hyperechogenic changes in lenticular nucleus of the brain in patients with cervical dystonia.

\section{Research Methodology}

The case-control study included patients with primary cervical dystonia and a control group of healthy volunteers. Patients were recruited from subjects treated between 2012 and 2017 in the Department of Neurology. All patients were diagnosed with primary cervical dystonia in accordance with the guidelines of European Federation of Neurological Society (EFNS) [17]. Healthy volunteers were recruited from inpatients and outpatients of our clinic and included neurologically normal persons, without signs and symptoms of movements or postural abnormalities, and without a history of neurological disease.

We excluded all individuals with a history of vascular, metabolic or traumatic injuries of central nervous system, patients with the history of perinatal health problems, those with abnormal neuroimaging results, patients with suspected drug-induced dyskinesia and those with opacity of temporal windows. One-hundred and eight patients were considered eligible for inclusion; 42 of them were subsequently excluded due to above-mentioned reasons. Thus, the study involved 66 patients and 71 controls. The study was conducted in accordance with the Helsinki Declaration and the study protocol was approved

${ }^{*}$ Corresponding author: Kubica AK, Medical University of Silesia, University Medical Center, Katowice, Poland, Tel: +48 3220836 00; E-mail: agukrzak@ gmail.com

Received March 19, 2018; Accepted April 26, 2018; Published April 30, 2018

Citation: Kubica AK, Ochudło S, Gawryluk J, Pawlicki K, Bar MR (2018) Hyperechogenicity of Lenticular Nuclei in Primary Cervical Dystonia. J Neurol Disord 6: 380. doi:10.4172/2329-6895.1000380

Copyright: (C) 2018 Kubica AK. This is an open-access article distributed under the terms of the Creative Commons Attribution License, which permits unrestricted use, distribution, and reproduction in any medium, provided the original author and source are credited. 
by the Local Ethics Committee. Each participant signed a consent form to participate in the study. The severity of dystonia was assessed clinically with the Toronto Western Spasmodic Torticollis Rating Scale (TWSTRS). Each patient had performed complete blood count, blood chemistry, as well as serum concentration of ceruloplasmin, ferritin, copper and iron. Either magnetic resonance imaging or computed tomography was also performed in each patient.

Ultrasound examinations were conducted during routine visits of patients who were treated with botulinum toxin within the treatment programme of the National Health Fund. Ultrasound assessment of subcortical brain structures was performed by a neurologist holding 10 years of experience in the field of transcranial ultrasound examinations and blinded to the relevant clinical data. The ultrasound examination was performed in a separate room with the use of Aloca Alpha Prosound 6 and according to the procedure recommended by the European Society of Neurosonology and Cerebral Hemodynamics of May 2004 [19,20].

The examination was carried out bilaterally through the parotid temporal windows determined by connecting the top of the orbit and the upper end of the auricle. A $2.3 \mathrm{MH}_{\mathrm{z}}$ probe was used, with a maximum penetration depth of $14-16 \mathrm{~cm}$ from the temporal bone window, so as to obtain the midbrain cross section (butterfly-shaped image) and the cross section of hyperechogenic adjacent structures of the basal cisterns. The obtained scan was enlarged twice and then, after manual selection, automatic ipsilateral measurement of the surface area of substantia nigra was performed. Hyperechogenicity of substantia nigra was defined as an area equal to or larger than $0.20 \mathrm{~cm}^{2}$ [20].

Similarly, in the horizontal projection of the third ventricle, a contralateral surface area measurement of hyperechogenic changes in the structures of the lenticular nucleus was made. Hyperechogenic changes were defined as areas of echogenicity which was similar or increased in comparison with the perimesencephalic cisterns [20]. Measurement of obtained hyperechogenic structures in the lenticular nucleus, as in the case of the substantia nigra, was selected manually, while surface area measurement was performed automatically.

\section{Statistical Analysis}

In order to select an adequate statistical test for the comparison of the studied groups, the Kolomogorov-Smirnov test will be performed. In cases where the test results indicated deviations from the normal distribution, the Mann-Whitney $\mathrm{U}$ test was used and a nonparametric alternative to the analysis of variance in the form of Kruskal-Wallis test with the Dunn post-hoc test was used. In cases when the test results indicate that the tested sample meets the conditions for normal distribution and homogeneity of variance, the student's t-test and ANOVA analysis were used, and also post-hoc type comparisons, if necessary: NIR test. The correlation strength was assessed using the $\mathrm{R}$ Sperman coefficient. The highest reliability credibility chi- square was also used.
Analyses were performed using the statistical software package, STATISTICA 13 Dell Inc. Results with P value $<0.05$ were considered statistically significant. Pearson's correlation coefficient was calculated to determine potential correlations between region of LN hyperechogenicity and duration of the disease, TWSTRS scale, the duration of treatment or the age of patients and between the width of third ventricle and the age of patients. Test $Z$ was used for 2 population proportions.

\section{Results}

Finally, from among the 108 patients, after excluding patients who did not meet the inclusion criteria, 66 patients with primary cervical dystonia were admitted to the study. The control group consisted of 71 people. The average age of patients was $52,84 \pm 14,45$, in the control group: $53,52 \pm 14,35$; range: $20-80$. The duration of the disease was $10,71 \pm 10,45$ years; women dominated in the material: $2,1: 1$ in the dystonia group and 1.6:1 in the control group (Table 1). No statistical differences were found between both groups $(\mathrm{p}=0.88)$. The clinical image of movement disorders assessed by means of the TWSTRS scale in the group of patients was on average $28.21(\mathrm{SD} \pm 13.59)$ and for individual components of the TWSTRS was as follows: Part I: 14.08 (SD \pm 5.93 ), Part II: 10.10 (SD \pm 6.71 ), Part III: 4.08 (SD \pm 3.86$)$. In the case of all patients, dystonia was stationary in nature and the observed fluctuations of severity of symptoms accounted for the effect of BTX treatment. The patients were treated with BTX for 5.8 years on average ( $\mathrm{SD} \pm 4.9$; range: $1-20$ years) and most of them showed significant improvement after BTX treatment.

Age and sex ratios of 66 patients and 71 controls, as well as clinical characteristics of dystonia (duration of the disease and severity of dystonia assessed with TWSTRS scale) are provided in Table 2. It was unilateral in majority of patients (75.76\%). Hyperechogenicity of the right lenticular nucleus was more often seen in patients with head rotation to the left $(p>0.01)$ but no other association between the direction of dystonic movement and location of changes was observed, although the hyperechogenicity contralateral to the direction of rotational dystonic movement was more common in absolute values. Hyperechogenic changes in lenticular nucleus were found in 8 controls (11.27\%); they were unilateral in all cases.

Average SN surface area in cervical dystonia was $0.10 \pm 0.08 \mathrm{~cm}^{2}$ on the right side and $0.09 \pm 0.05 \mathrm{~cm}^{2}$ on the left side, and average $\mathrm{SN}$ surface area in control group was $0.08 \pm 0.03 \mathrm{~cm}^{2}$ on both side ( $\left.\mathrm{p}>0.005\right)$. The surface area of the hyperechogenic changes was respectively $0.10 \pm 0.12$ $\mathrm{cm}^{2}$ on the right side and $0.05 \pm 0.10 \mathrm{~cm}^{2}$ on the left side and they were significantly different from the changes observed in the control group with $0.004 \pm 0.02 \mathrm{~cm}^{2}$ on the right side and $0.005 \pm 0.025 \mathrm{~cm}^{2}$ on the left side (Table 3).

\begin{tabular}{|c|c|c|}
\hline Variables & Patient with cervical dystonia (n=66) & Control group (n=71) \\
\hline Age (years); mean (SD) & $52.84(14.45)$ & $53.52(14.04)^{*}$ \\
\hline Sex women/men (n) & $45 / 21$ & $60 / 11^{*}$ \\
\hline Disease duration (years); mean (SD) & $10.71(10.45)$ \\
\hline Peverity of dystonia (TWSTRS score); mean (SD) Total & $28.21(13.58)$ \\
\hline Part II & $14.08(5.9)$ & - \\
\hline Part III & $10.10(6.71)$ & - \\
\hline SD: Standard Deviation; TWSTRS: Toronto Western Spasmodic Torticollis Rating Scale $p>0.005$. \\
\hline
\end{tabular}

Table 1: Comparison of age and sex between patients with cervical dystonia and controls as well as clinical characteristics of dystonia in studied patients. 


\begin{tabular}{|c|c|c|c|c|c|c|}
\hline Hyperechogenicity & $\begin{array}{l}\text { Dystonic patient with head } \\
\text { rotation to right }(n=23)\end{array}$ & $\begin{array}{l}\text { Dystonic patient with head } \\
\text { rotation to the left }(n=43)\end{array}$ & $\begin{array}{l}\text { p- value } \\
\text { (test Z) }\end{array}$ & $\begin{array}{l}\text { All patient with cervical } \\
\text { dystonia }(n=66)\end{array}$ & $\begin{array}{c}\text { Control group } \\
(n=71)\end{array}$ & $\begin{array}{l}\text { p-value } \\
\text { (test Z) }\end{array}$ \\
\hline Right lenticular nucleus & $12(52.17)$ & $24(55.81)$ & 0.77 & $36(54.55)$ & $4(5.63)$ & $<0.0001$ \\
\hline Left lenticular nucleus & $9(39.13)$ & $13(30.23)$ & 0.46 & $22(33.33)$ & $4(5.63)$ & $<0.0001$ \\
\hline Both lenticular nuclei & $5(21.73)$ & $3(6.97)$ & 0.079 & $8(12.12)$ & 0 & $<0.0001$ \\
\hline Any location & $17(73.91)$ & $33(76.74)$ & 0.79 & $50(75.76)$ & $8(11.27)$ & $<0.0001$ \\
\hline None & $6(26.09)$ & $10(23.25)$ & 0.79 & $16(24.24)$ & $63(88.73)$ & $<0.0001$ \\
\hline
\end{tabular}

Test Z for 2 population proportions.

*Data shown as number (\%).

Table 2: Prevalence of hyperechogenic changes among patients with cervical dystonia (including patients with head rotation either to the left or to the right) and control subjects. Hyperechogenicity of the lenticular nucleus was found more often in patients with cervical dystonia than in controls.

\begin{tabular}{|c|c|c|c|c|c|}
\hline \multirow{2}{*}{ Variables } & \multicolumn{2}{|c|}{ Patient with cervical dystonia $(n=66)$} & \multicolumn{2}{|c|}{ Control group $(n=71)$} & \multirow{2}{*}{ P-value } \\
\hline & Mean & SD & Mean & SD & \\
\hline Age (years) & 52.84 & 14.44 & 53.52 & 14.03 & 0.88 \\
\hline Left substantia nigra $\left(\mathrm{cm}^{2}\right)$ & 0.09 & 0.05 & 0.08 & 0.03 & 0.10 \\
\hline Right substantia nigra $\left(\mathrm{cm}^{2}\right)$ & 0.10 & 0.08 & 0.08 & 0.03 & 0.43 \\
\hline Third ventricle width (mm) & 4.7 & 1.48 & 4.1 & 1.29 & 0.48 \\
\hline Hyperechogenicity of left lenticular nucleus $\left(\mathrm{cm}^{2}\right)$ & 0.05 & 0.10 & 0.005 & 0.02 & 0.008 \\
\hline Hyperechogenicity of right lenticular nucleus $\left(\mathrm{cm}^{2}\right)$ & 0.10 & 0.12 & 0.004 & 0.019 & 0.000029 \\
\hline
\end{tabular}

U-Mann test

Table 3: Measurement of the surface of the substantia nigra. surface of changes in the lenticular nucleus and the width of the third ventricles in patients with cervical dystonia and in the control group.

Table 3 provides data on surface areas of lenticular nuclei and substantia nigra, as well as on third ventricle width among patients with cervical dystonia (including subgroups of men and women) and in control group. Surface area of the lenticular nuclei, either left or right, was greater in patients with dystonia than in controls. The surface area of the substantia nigra in one control subject was a boderline value $\left(0.20 \mathrm{~cm}^{2}\right)$. This person appeared to have a positive family history of neurodegenerative diseases. Measurements of the width of the third ventricle showed no significant difference between the groups ( $\mathrm{p}=0.48$ ). No correlation was found between hyperechogenicity of lenticular nucleus and the duration of the disease, the severity of dystonia measured with the TWSTRS scale, the duration of treatment or the age of patients. The width of third ventricle correlated with the age of patients $(r=0.39 ; \mathrm{p}<0.01)$.

\section{Discussion}

We have shown that hyperechogenic changes in the lenticular nucleus are more prevalent in patients with primary cervical dystonia than in healthy controls. The change was not related with severity, duration of dystonia and any correlation was between hyperechogenicity of LN and duration of botulinum toxin treatment. Similar results were also reported by other investigators [4,21,22]. This observation has important diagnostic implications because, as in PD, the performance of a simple and widely available TCS test might be useful adjunct to clinical diagnosis, which so far has been based on clinical observations and negative results of additional tests [23,24]. Recent technological progress in the quality of the ultrasound image allows for the more precise measurement of abnormalities deep within the brain structure. In 2010, Gaenslen described unspecified hyperechogenicity of the lenticular nuclei in more than $75 \%$ of patients with cervical dystonia, in more than half of patients with other focal dystonias and very rarely in patients with facial dystonia and genetically determined dystonia [21]. Other researchers confirmed more frequent occurrence of ultrasound TCS changes of subcortical structures also in other neurodegenerative diseases: multi-system atrophy, essential tremor, progressive supranuclear palsy, corticobasal degeneration, neurodegeneration associated with accumulation of iron, Huntington disease, Wilson disease, dementia with Lewy bodies, some spinocerebellar ataxias and restless legs syndrome [23,25-28]. We did not find a correlation between the size of the observed changes and dystonia severity or duration of the disease. The only correlation was the association between the width of the third ventricle and age of patients, which may correspond to the progression of regressive changes of the brain associated with aging.

Recent studies with the use of magnetic resonance imaging (MRI), specifically with diffusion tensor imaging, revealed the presence of changes in the basal ganglia, thalamus, motor and premotor cortex, cerebellum, frontal cortex, temporal cortex and parietal region, which may be related to the hyperechogenic changes observed in TCS examination in cervical dystonia [6]. Volumetric MRI scans (voxel-based morphometry) revealed the presence of structural changes in dystonia: volume increase of the gray matter of the inner part of the globus pallidus, thalamus, cerebellum, primary motor cortex, and supplementary motor cortex $[29,30]$. Reported findings are inconsistent, however, because the reduction of the gray matter volume was also noted in the putamen, supplementary motor cortex, right primary visual cortex and right dorsolateral premotor cortex [31-33]. Diffusion tensor imaging delineates pathology of white matter fibres, showing both macrostructural changes (diffusivity, as shown in apparent diffusion coefficient [ADC]) and alterations of fibres organisation (fractional anisotropy [FA]). In patients with inherited forms of dystonia, some studies showed reduction of FA in sensorimotor cortex, corpus callosum and increase in the putamen. Another study showed reinforcement of diffusivity (ADC) within the striatum, globus pallidus and caudate nucleus in dystonia.

Magnetisation transfer imaging detects relative proportions of free protons and protons associated with macromolecules, showing changes in the myelin and neuronal loss. These multifocal changes in structural neuroimaging support the hypothesis that dystonia is one of the circuits disorders of the basal ganglia resulting from pathologic disturbances in neuronal activity throughout specific cortico-subcortical loops [7] but cannot show any marker specific for the diagnosis. The cause of lenticular nucleus hyperechogenicity in primary dystonia is unclear. However, post-mortem measurements of trace metals and calcium were performed in brain tissue of 3 patients with adult-onset idiopathic 
dystonia and 10 healthy controls and showed increased level of copper and manganese in the dystonic patient compared with controls, whereas there was no difference in the levels of iron, zinc, or calcium [13]. Further analyses revealed that the level of the copper-metabolizing Menkes protein was reduced, which could lead to increased levels of copper in patients with primary dystonia. A subsequent study showed decreased levels of mRNA for Menkes protein and reduced copper concentrations in peripheral blood lymphocytes in patients with cervical dystonia compared with patients with blepharospasm and healthy controls [15]. This study suggests an alteration in copper metabolism in some forms of dystonia.

In 2005, Walter et al. [13] noted the presence of hyperechogenic changes in the lenticular nucleus in 19 out of 21 patients with Wilson disease, i.e., the condition with proven pathomechanism of copper metabolism disorder. This hyperechogenicity of the lenticular nucleus appears as a dot and resembles the TCS findings in idiopathic dystonia [13]. In patients with Wilson disease associated with neurological manifestation, the area of hyperechogenicity within the lenticular nucleus is more extensive and correlates with disease severity, which enables differential diagnosis of the metabolic disorders [34]. It is probable that the hyperechogenic TCS changes in cervical dystonia are also associated with excessive accumulation of copper [13-15], which might contribute to the pathogenesis of dystonia as a modulator of synaptic function and neurotransmission. This early hypothesis requires further research, also because some studies do not confirm those observations [35]. Our study, along with the previous ones, indicates a more frequent occurrence of hyperechogenic changes in lenticular nuclei in primary cervical dystonia $[22,35]$. We still do not know whether they differentiate the type of dystonia and if they are associated with disease duration or depend on the time and method of treatment. Perhaps their presence is a marker for the risk of developing dystonia, which may be indicated by the observed presence of hyperechogenic TCS changes in one healthy excluded subject with a positive medical history towards dystonia. These questions, and the observed high prevalence of changes within the basal ganglia, warrant continued research. Undoubtedly, a weak point of the study might be not a very large group of the patients and the ultrasounds were performed by only one physician.

\section{Conclusion}

Beyond the subjective image perception, the impact on results might be performance of neuroimaging not at the same time as ultrasound. We suggest that the observed ultrasound basal ganglia hyperechogenicity in primary dystonia are associated with the pathogenesis of the disease, and their location, taking the complex pathophysiological mechanism of the disease into account, may be limited not only to the lenticular nucleus and substantia nigra. It is likely that they also appear in other brain structures; a thorough examination will allow us to determine the different characteristic patterns for different types of dystonia. In conclusion, we suggest that the hyperechogenic changes within the lenticular nuclei are prevalent in patients with primary cervical dystonia and are an important diagnostic clue.

\section{References}

1. Geyer HL, Bressman SB (2006) The diagnosis of dystonia. Lancet Neurol 5 : 780-790

2. Hallet M (2011) Neurophysiology of dystonia: the role of inhibition. Neurobiol Dis 42: $177-184$.

3. Lehericy S, Tijsen MA, Vidaihert M, Kaji R, Meunier S (2013) The anatomica basis of dystonia: Current view using neuroimaging. Mov Disord 28: 944-957.
4. Bathia KP, Marsden CD (1994) The behavioral and motor consequences of focal lesions of the basal ganglia in man. Brain 117: 859-876.

5. Argyelan M, Carbon M, Niethammer M, Ulug AM, Voss HU, et al. (2009) Cerebellothalamocortical connectivity regulates penetrance in dystonia. J Neurosci 29: 9740-9747.

6. Prell T, Peschel T, Kohler B, Bokemeyer MH, Dengler R, et al. (2013) Structural brain abnormalities in cervical dystonia. BMC Neurosci 16: 14-123.

7. DeLong MR, Wichmann T (2007) Circuits and circuit disorders of the basal ganglia. Arch Neurol 64: 20-24.

8. Vitek JL (2002) Pathophysiology of dystonia: A neuronal model. Mov Disord 17: S49-62.

9. Berrardelli A, Rothwell JC, Hallet M, Thompson PD, Manfredi M, et al. (1998) The pathophysiology of primary dystonia. Brain 121: 1195-212.

10. Nauman M, Becker G, Tikya KV, Supprian T, Reiners K (1996) Lenticular nucleus lesion in idiopatic dystonia detected by transcranial sonography. Neurology 47: 1284-1290.

11. Tewari A, Fremont R, Khodakhak K (2017) It's not just the basal ganglia: cerebellum as a target for dystonia therapeutics. Mov Disord 32: 1537-1545.

12. Defazio G, Berardelli A, Hallett M (2007) Do primary adult-onset focal dystonias share aetiological factors? Brain 130: 1183-1193.

13. Becker G, Berg D, Rausch WD, Lange HK, Riederer P, et al. (1999) Increased tissue copper and manganese content in the lentiform nucleus in primary adultonset dystonia. Ann Neurol 46: 260-263

14. Berg D, Weishaupt A, Francis MJ, Miura N, Yang XL, et al. (2000) Changes of cooper-transporting proteins and ceruloplasmin in the lentiform nuclei in primary adult-onset dystonia. Ann Neurol 47: 827-830.

15. Kruse N, Berg D, Francis MJ, Naumann M, Rausch WD, et al. (2001) Reduction in Menkes mRNA and cooper in leukocytes of patients with primary adult-onse dystonia. Ann Neurol 49: 405-408.

16. Alberto A, Bhatia K, Bressman SB (2013) Phnomenology and classification of dystonia: A consensus update. Mov Disord 28: 863-873.

17. Albanese A, Asmus F, Bhatia KP, Elia AE, Elibol B, et al. (2011) EFNS guidelines on diagnosis and treatment of primary dystonias. Eur $\mathrm{J}$ Neurol 18: 5-18.

18. Becker G, Seufert J, Bogdahn U, Reichmann H, Reiners K (1995) Degradation of substantia nigra in chronic Parkinson's disease visualized by transcrania color-coded real-time sonography. Neurology 45: 182-184.

19. Bartova P, Skoloudik D, Bar M (2008) Transcranial sonography in movement disorders. Biomed pap Med Fac Univ Palacky Olomounc Czech Rep 152 251-258.

20. Walter U, Skouloudik D (2014) Transcranial sonography (TCS) of brain parenchyma in movement disorders: quality standards, diagnostic applications and novel technologies. Ultraschall Med 35: 322-331.

21. Gaensler A (2010) Transcranial sonography in dystonia. Int Rev Neurobiol 90 179-187.

22. Zhang Y, Zhang YC, Sheng YJ (2016) Sonographic alteration of basal ganglia in different forms of primary focal dystonia: A cross-sectional study. Chin Med $\mathrm{J}(\mathrm{EN})$ 129: 942-945

23. Walter U, Dressler D, Wolters AIW (2006) Sonographic discrimination of dementia with Lewy bodies and Parkinsons disease with dementia. J. Neurol 253: 448-454.

24. Behnke S, Eyding J, Niehaus L, Postert T, Seidel G, et al. (2007) Transcrania brain parenchyma sonography in movement disorders: state of the art. Ultrasound Med Biol 33: 15-25.

25. Dexter DT, Jenner P, Schapira AHV, Marsden CD (1992) Alteretion in levels of Iro, ferritin, and other trace metals in neurodegenerative disease affecting the basal ganglia. Ann Neurol 32: S94-S100.

26. Mijajlovic MD, Tsivgoulis G, Sternic N (2014) Transcranial brain parenchymal sonography in neurodegenerative and psychiatric disease. J Ultrasound Med 33: 2061-2068.

27. Poster T, Lack B, Kuhn W, Jergas M, Andrich J, et al. (1999) Basal ganglia alterations and brain atrophy in Huntington's disease depicted by transcrania real time sonography. J Neuro Neurosurg Psychiatry 67: 457-462.

28. Godau J, Schwietzer KJ, Liepelt I, Gerloff C, Berg D (2007) Substantia nigra 
Citation: Kubica AK, Ochudło S, Gawryluk J, Pawlicki K, Bar MR (2018) Hyperechogenicity of Lenticular Nuclei in Primary Cervical Dystonia. J Neurol Disord 6: 380. doi:10.4172/2329-6895.1000380

Page 5 of 5

hyperechogenicity: definition and findings in restless legs syndrome. Mov Disord 22: 187-192.

29. Becker G, Berg D, Francis M, Naumann M (2001) Evidence for disturbances of copper metabolism indystonia: From the image towards a new concept. Neurology 57: 2290-2294

30. Egger K, Mueller J, Schocke M, Brenneis C, Rinnerthaler M, et al. (2007) Voxel based morphometry reveals specific gray matter changes in primery dystonia. Mov Disord 22: 1538-1542.

31. Draganski B, Thun-Hohenstein C, Bogdahn U, Winkler J, May A (2003) "Motor circuit" gray matter changes in idiopathic cervical dystonia. Neurology 61 : 1228-1231.
32. Obermann M, Yaldizli O, De Greiff A, Lachenmayer ML, Buhl AR, et al. (2007) Morphometric changes of sensorimotor structures in focal dystonia. Mov Disord 22: $1117-1123$.

33. Prell T, Peschel T, Kochler B, Bokemeyer MH, Dengler R, et al. (2013) Structural brain abnormalities in cervical dystonia. BMC Neuroscience 14: 123 .

34. Walter U, Krolikowski K, Tarnacka B, Benecke R, Czlonkowska A, et al (2005) Sonographic detection of basal ganglia lesions in asymptomatic and symptomtic Wilsons disease. Neurology 64: 1726-1732.

35. Hagenah J, König IR, Kötter C, Seidel G, Klein C, et al. (2011) Basal ganglia hiperechogenicity does not distinguish between patient with primary dystonia and healthy individuals. J Neurol 258: 590-595. 